\title{
EQUIVALENCE OF METHODS FOR EVALUATION OF SEQUENCES
}

RALPH PALMER AGNEW

1. Introduction. A matrix $a_{n k}$ of real or complex constants determines a transformation

$$
\sigma_{n}=\sum_{k=0}^{n} a_{n k} s_{k}, \quad n=0,1,2, \cdots
$$

by which a sequence $s_{n}$ is evaluable to $\sigma$ if $\sigma_{n} \rightarrow \sigma$ as $n \rightarrow \infty$. The transformation is said to be regular, or to include convergence, if $\lim \sigma_{n}$ $=\lim s_{n}$ whenever $\lim s_{n}$ exists. We shall use the simple and well known Silverman-Toeplitz necessary and sufficient conditions for regularity of (1.1), namely

$$
\begin{aligned}
\sum_{k=0}^{n}\left|a_{n k}\right| & \leqq C, & n=0,1,2, \cdots, \\
\lim _{n \rightarrow \infty} a_{n k} & =0, & k=0,1,2, \cdots, \\
\lim _{n \rightarrow \infty} \sum_{k=0}^{n} a_{n k} & =1 . &
\end{aligned}
$$

For expositions of the subject, see Hardy [4] and Cooke [3]; our terminology agrees with that of Hardy.

Unfortunately, there are no such simple necessary and sufficient conditions that (1.1) be included by convergence, that is, be such that $\lim s_{n}=\lim \sigma_{n}$ whenever $\lim \sigma_{n}$ exists. Some Mercerian theorems, which are treated briefly by Hardy [4], solve the problem for special classes of transformations involving a parameter in a simple way, but the general problem never has been and perhaps cannot be successfully attacked. It is the object of this note to prove the following theorem which gives simple sufficient conditions that (1.1) be included by convergence, and to show that the conclusion will fail to follow if the condition (1.31) or any one of the three conditions for regularity is removed from the hypothesis.

THEOREM 1.3. If (1.1) is regular and if

$$
\liminf _{n \rightarrow \infty}\left[\left|a_{n n}\right|-\sum_{k=0}^{n-1}\left|a_{n k}\right|\right]>0 \text {, }
$$

Received by the editors December 13, 1951. 
then (1.1) is included by convergence.

It is a consequence of this theorem that if (1.1) is regular and satisfies (1.31), then (1.1) is equivalent to convergence. The hypotheses of Theorem 1.3 imply that

$$
\liminf _{n \rightarrow \infty}\left|a_{n n}\right| \geqq(1+L) / 2
$$

where $L$ is the left member of (1.31), and hence that $a_{n n} \neq 0$ for each sufficiently great $n$. If it happens that $a_{n n} \neq 0$ for each $n$, then the matrix $A$ in (1.1) has a unique inverse $A^{-1}$ which is also triangular. In this case the conclusion of Theorem 1.3 implies that the inverse $A^{-1}$ must be regular.

We shall refer to the following theorem which is at least superficially less general than Theorem 1.3.

THEOREM 1.4. If (1.1) is regular and if

$$
\lim _{n \rightarrow \infty}\left[\left|a_{n n}\right|-\sum_{k=0}^{n-1}\left|a_{n k}\right|\right]>0,
$$

then (1.1) is equivalent to convergence.

Theorem 1.3 includes three somewhat less general theorems obtained by the author [1] to show equivalence of the arithmetic mean transformation

$$
M_{n}=\frac{1}{n} \sum_{k=1}^{n} s_{k}
$$

and such transformations as

$$
\begin{gathered}
H_{n}=\sum_{k=1}^{n} \frac{1}{\log 2} \frac{1}{n+k} s_{k} \\
H_{n}^{\prime}=\sum_{k=1}^{n} \frac{1}{\log 2} \frac{1}{2 n-k+1} s_{k} .
\end{gathered}
$$

The latter problem was suggested to the author by Einar Hille. Theorem 1.4 was given by Mazur and Orlicz [6] in a note containing no proofs. It seems that these authors were somewhat generous in referring to Theorem 1.4 as "un théorème de M. R. Agnew" [1]; while Theorems 1.3 and 1.4 and methods of proof are strongly suggested by [1], they do not appear in [1]. We shall point out in $\$ 2$ a manner in which one can supply the details of the argument of Mazur and Orlicz [6] that Theorem 1.4, and Theorem 1.3 as well, 
are corollaries of one of their fundamental theorems which we state:

LEMMA 1.6. If (1.1) is regular and if at least one divergent sequence is evaluable (1.1), then at least one unbounded sequence is evaluable (1.1).

The fact that Theorem 1.4 is a useful theorem may be seen in [5] where Karamata made implicit use of a special case and in [2] where several explicit applications were needed. $\$ 5$ shows that nontriangular transformations escape theorems analogous to Theorem 1.3 .

2. Proof of Theorem 1.3. We prove Theorem 1.3 by showing that if $s_{n}$ is a divergent sequence, then $\sigma_{n}$ must also be divergent. Suppose first that $s_{n}$ is unbounded. Then for an infinite set of values of $n$

$$
\left|s_{k}\right| \leqq\left|s_{n}\right|, \quad k=0,1,2, \cdots, n-1 \text {. }
$$

For such values of $n$

$$
\begin{gathered}
\left|\sigma_{n}\right|=\mid a_{n n} s_{n}+ \\
+\sum_{k=0}^{n-1} a_{n k} s_{k}|\geqq| a_{n n}|| s_{n}\left|-\sum_{k=0}^{n-1}\right| a_{n k}|| s_{k} \mid \\
\geqq\left[\left|a_{n n}\right|-\sum_{k=0}^{n-1}\left|a_{n k}\right|\right]\left|s_{n}\right|
\end{gathered}
$$

Therefore $\lim \sup \left|\sigma_{n}\right|=\infty$ and $\sigma_{n}$ is divergent. Thus we have shown, by a very simple argument that appears in $[1$, p. 247], that (1.1) can evaluate no unbounded sequences. It follows from Lemma 1.6 of Mazur and Orlicz that (1.1) can evaluate no divergent sequences and Theorem 1.3 is proved.

The above proof shows that (1.31) alone, without any of the three conditions for regularity, is sufficient to imply that (1.1) can evaluate no unbounded sequences. The conditions for regularity are used in the above proof of Theorem 1.3 only when the argument of Mazur and Orlicz was applied. All this leaves the possibility that one could modify our direct attack on unbounded sequences to show directly that (1.31) alone is sufficient to imply that (1.2) can evaluate no bounded divergent sequences.

3. Bounded sequences. We now give a detailed proof showing how each of the three conditions for regularity enters into a direct demonstration of the fact that a regular transformation (1.1) satisfying (1.31) can evaluate no bounded divergent sequences; this proof motivates the examples of $\$ 4$.

Let $s_{n}$ be a bounded divergent sequence of complex numbers. Let $\sigma$ be any complex number and let $R=\lim \sup \left|s_{n}-\sigma\right|$. Then $R>0$ and using (1.23) gives 


$$
\sigma_{n}-\sigma=o(1)+a_{n n}\left(s_{n}-\sigma\right)+\sum_{k=0}^{n-1} a_{n k}\left(s_{k}-\sigma\right) .
$$

Letting $\epsilon>0$ and choosing $k_{0}$ such that $\left|s_{k}-\sigma\right|<R+\epsilon$ when $k>k_{0}$, we use (1.22) to obtain

$$
\left|\sigma_{n}-\sigma\right| \geqq o(1)+\left|a_{n n}\right|\left|s_{n}-\sigma\right|-\sum_{k=k_{0}}^{n-1}\left|a_{n k}\right|(R+\epsilon) .
$$

Use of (1.21) then gives

$$
\left|\sigma_{n}-n\right| \geqq o(1)-\epsilon C+\left|a_{n n}\right|\left|s_{n}-\sigma\right|-\sum_{k=0}^{n-1}\left|a_{n k}\right| R .
$$

For an increasing sequence of values of $n$, we have $\left|s_{n}-\sigma\right|>R-\epsilon$. For such values of $n$ we have

$$
\left|\sigma_{n}-\sigma\right| \geqq o(1)-2 \epsilon C+\left[\left|a_{n n}\right|-\sum_{k=0}^{n-1}\left|a_{n k}\right|\right] R
$$

and for all sufficiently great such values of $n$ we have

$$
\left|\sigma_{n}-\sigma\right| \geqq o(1)-2 \epsilon C+(L-\epsilon) R
$$

where $L$ is the left member of (1.31). Therefore $\lim \sup \left|\sigma_{n}-\sigma\right|$ $\geqq-2 \epsilon C+(L-\epsilon) R$. Since this holds for each $\epsilon>0$, we must have lim sup $\left|\sigma_{n}-\sigma\right| \geqq L R>0$. Since this holds for each complex number $\sigma$, the sequence $\sigma_{n}$ must be divergent. Thus we have a direct proof which shows that we can quite easily avoid use of the Mazur-Orlicz theorem. It should be remarked, however, that the above proof is a little less involved than the proofs previously given by the author [1] for the special cases, and that the idea of Mazur and Orlicz that they should use their theorem in this connection is very elegant.

4. Examples. That the conclusion of Theorem 1.3 would fail if (1.31) were removed from the hypothesis is obvious; the arithmetic mean transformation (1.5) which evaluates the divergent sequence $s_{n}=(-1)^{n}$ is one of countless classic examples. The matrix $a_{n k}$ for which $a_{n n}=(-1)^{n}$ and $a_{n k}=0$ otherwise satisfies (1.31) and all of the conditions for regularity except (1.23). It evaluates the divergent sequence $s_{n}=(-1)^{n}$. The matrix $a_{n k}$ for which $a_{n n}=1, a_{n, 0}=-a_{n, 1}$ $=(-1)^{n+1} / 3$ when $n \geqq 3$, and $a_{n k}=0$ otherwise, satisfies (1.31) and all of the conditions for regularity except (1.22). It evaluates the divergent sequence for which $s_{0}=3, s_{1}=0$, and $s_{n}=(-1)^{n}$ when $n>1$.

That a matrix satisfying (1.31) and all of the conditions for regularity except (1.21) could evaluate no bounded divergent sequences 
seems more plausible, but the proposition escapes proof and the following example shows that it is false. Let $a_{n n}=1+n^{2} / 2, a_{n, n-1}=0$, $a_{n, n-2}=-n^{2} / 2$, and $a_{n k}=0$ when $k<n-2$. The divergent sequence $s_{n}=(-1)^{n}[1+1 /(n+1)]$ is evaluable to 0 by this matrix. The matrix $a_{n n}=(n+1)^{2}, a_{n, n-1}=n(n+2)$, and $a_{n k}=0$ otherwise also evaluates this sequence.

5. Nontriangular matrices. The transformation (1.1) which we have heretofore considered has the form

$$
\sigma_{n}=\sum_{k=0}^{\infty} a_{n k} s_{k}, \quad n=0,1,2, \cdots,
$$

where $a_{n k}=0$ when $k>n$, the matrix $a_{n k}$ being triangular. We remove the requirement that $a_{n k}=0$ when $k>n$, and replace (1.31) by

$$
\liminf _{n \rightarrow \infty}\left[\left|a_{n n}\right|-\sum_{k=0}^{\infty} *\left|a_{n k}\right|\right]>0
$$

where the star on the $\sum$ signifies that the term for which $k=n$ is to be omitted from the sum. A trivial modification of the proof in $\$ 3$ gives the following theorem.

THEOREM 5.3. If (5.1) is regular and satisfies (5.2), then no bounded divergent sequences are evaluable (5.1).

On the other hand, the argument in (2.21) breaks down completely if $a_{n k} \neq 0$ when $k>n$. A very interesting example of a regular transformation of the form (5.1) which satisfies (5.2) and in fact much stronger significant conditions such as $a_{n k} \geqq 0, a_{n k}=0$ when $k>n+1, \lim a_{n n}=1$, and $\lim \sum *\left|a_{n k}\right|=0$, and which nevertheless evaluates some (necessarily unbounded) divergent sequences, is

$$
\sigma_{n}=\left(1-\frac{1}{n+3}\right) s_{n}+\frac{1}{n+3} s_{n+1}, \quad n=0,1,2, \cdots .
$$

This transformation evaluates the divergent sequence $s_{n}$ $=(-1)^{n}(n+1) !$.

The following remarks concerning the transformation (5.4) are of interest in connection with the theory of infinite matrices; see Cooke [3] where the subject of this paper is not treated but many instructive examples are given on p. 169 and elsewhere. Let $A=\left(a_{n k}\right)$ denote the matrix of (5.4) so that $a_{n n}=1-1 /(n+3), a_{n, n+1}=1 /(n+3)$, and $a_{n k}=0$ otherwise. Let $B=\left(b_{n k}\right)$ denote the matrix for which $b_{n n}=1 /(n+3), b_{n, n+1}=-1 /(n+3)$, and $b_{n k}=0$ otherwise. Let $I$ denote the identity matrix, and let $\lambda=1$. Then 


$$
A=I-\lambda B .
$$

The norm of $B$, defined to be the least upper bound of the numbers $\sum_{k=0}^{\infty}\left|b_{n k}\right|$, is $2 / 3$. It follows by very well known arguments that the series in the right member of

$$
A^{-1}=I+\lambda B+\lambda^{2} B^{2}+\lambda^{3} B^{3}+\cdots
$$

converges to a matrix $A^{-1}$ which is an inverse of $A$ in the sense that $A A^{-1}=A^{-1} A=I$. Moreover this inverse $A^{-1}$ is the matrix of a regular transformation. If a convergent sequence $\sigma$ is the transform of a bounded sequence $s$ so that $\sigma=A s$, then we can use the argument $A^{-1} \sigma=A^{-1}(A s)=\left(A^{-1} A\right) s=s$ to conclude that the sequence $s$ must be convergent; but the conclusion fails in case $s_{n}=(-1)^{n}(n+1)$ ! and it must therefore be untrue that, in this case, $A^{-1}(A s)=\left(A^{-1} A\right) s$. Another peculiarity of the transformation (5.4) lies in the fact that its matrix $A$ has an inverse $A^{-1}$; but nevertheless one can, when a sequence $\sigma_{0}, \sigma_{1}, \cdots$ is given, assign an arbitrary value to $s_{0}$ and then use the equation (5.4) one by one to determine uniquely the remaining elements $s_{1}, s_{2}, \cdots$ of the sequence $s_{n}$ so that (5.4) holds. Thus, even for a regular nontriangular matrix satisfying very restrictive conditions, existence of a regular inverse of the matrix $A$ in (5.1) does not imply unique solvability of the equations (5.1) for $s_{0}, s_{1}, \ldots$ when the sequence $\sigma_{0}, \sigma_{1}, \cdots$ is given, and does not imply that (5.1) is equivalent to convergence.

The inverse matrix $A^{-1}$ defined by (5.42) has a quite simple form; both of the matrices $A$ and $A^{-1}$ of the example are displayed below.

$(A)$

$$
\begin{array}{ccccccc}
\frac{2}{3} & \frac{1}{3} & 0 & 0 & 0 & 0 & \ldots \\
0 & \frac{3}{4} & \frac{1}{4} & 0 & 0 & 0 & \cdots \\
0 & 0 & \frac{4}{5} & \frac{1}{5} & 0 & 0 & \cdots \\
0 & 0 & 0 & \frac{5}{6} & \frac{1}{6} & 0 & \cdots \\
0 & 0 & 0 & 0 & \frac{6}{7} & \frac{1}{7} & \cdots \\
0 & 0 & 0 & 0 & 0 & \frac{7}{8} & \cdots
\end{array}
$$




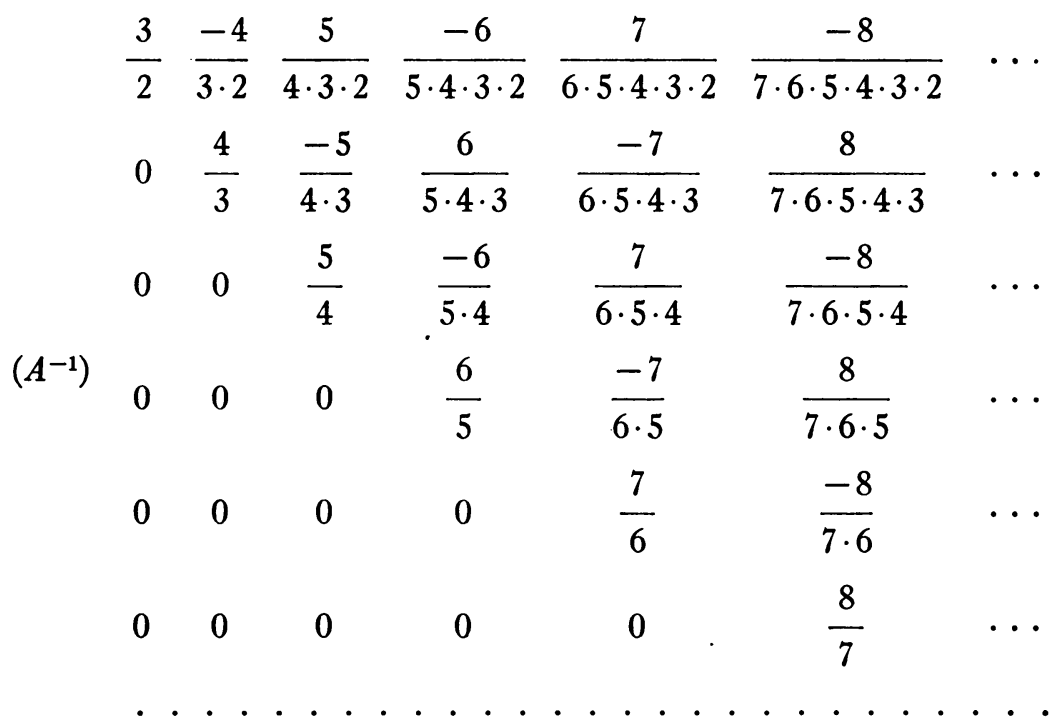

One who cares to do so can easily supplement or replace the analytic considerations involving (5.42) by direct manipulation with the displayed matrices to see that the matrix $A$ possesses an inverse $A^{-1}$ for which $A A^{-1}=A^{-1} A=I$ and to see that $A^{-1}$ is regular.

\section{REFERENCES}

1. R. P. Agnew, On equivalence of methods of evaluation of sequences, Tôhoku Math. J. vol. 35 (1932) pp. 244-252.

2. - Rogosinski-Bernstein trigonometric summability methods and modified arithmetic means, Ann. of Math. (to appear).

3. R. G. Cooke, Infinite matrices and sequence spaces, London, 1950.

4. G. H. Hardy, Divergent series, Oxford, 1949.

5. Jovan Karamata, Über die Beziehung zwischen dem Bernsteinschen und Cesdroschen Limitierungsverfahren, Math. Zeit. vol. 52 (1949) pp. 305-306.

6. Stanislaw Mazur and Wladyslaw Orlicz, Sur les méthodes linéaires de sommation, C. R. Acad. Sci. Paris vol. 196 (1933) pp. 32-34.

Cornell University 